\title{
Percutaneous nephrostomic decortication: a microinvasive surgery for posterior renal cyst
}

\author{
Hequn Chen, Yang Li, Feng Zeng, Cheng He, Yu Cui, Jinbo Chen, Huimin Zeng, Siqi Lu, Weiguo Wang, \\ Zhiyong Chen
}

Department of Urology, Xiangya Hospital, Central South University, Changsha, China

Correspondence to: Zhiyong Chen. 87 Xiangya Road, Urology Department, Xiangya Hospital, Changsha, China. Email: jeffastone@126.com.

\begin{abstract}
To assess the safety, feasibility, and efficacy of a modified process we developed in order to perform decortication surgeries for simple renal cysts through a percutaneous approach, 18 patients with simple renal cysts larger than $5 \mathrm{~cm}$ were treated with our new technique, from November 2016 to January 2019. All of the cysts were evaluated with ultrasonography and contrast-enhanced computed tomography. The Surgical procedure: as the standard mini-Percutaneous Nephrostomy procedure, a puncture was made directly into the cyst through the cyst roof under ultrasound guidance. After the inner cyst wall examination, the sheath was then retracted to just outside the cyst roof. The sheath together with the scope was used to detach the roof from the perirenal fat, after which the cyst roof was resected using a laser. A drain tube was left in the retroperitoneal space for 1-2 days. Subsequently, all patients were discharged 1 to 2 days postsurgery. Skin incision was less than $1 \mathrm{~cm}$. No major complication was observed. The follow-up time ranges from 14 to 37 months. One case had an incompletely resected cyst with the cyst volume being decreased by more than $60 \%$. This patient didn't receive any additional treatments during his follow-up. For all the other patients, the cysts had completely disappeared and no relapse had occurred. In conclusion, in the selected patients with a simple posterior renal cyst, our new technique is a safe and an effective option. It is also considered the least invasive decortication surgery for posterior renal cyst.
\end{abstract}

Keywords: Renal cyst; decortication; percutaneous; surgery; ultrasound

Submitted Apr 20, 2020. Accepted for publication Oct 28, 2020.

doi: $10.21037 /$ tau-20-875

View this article at: http://dx.doi.org/10.21037/tau-20-875

\section{Introduction}

The prevalence of renal cysts in the general population is around $20 \%$ to $50 \%$ and increases with age (1-3). Most renal cysts are diagnosed incidentally without any symptoms and grow slowly. But some cysts could grow to very large ones, causing pain, hematuria, hypertension, renal parenchymal compression and sometimes even up to the point of obstructing the collecting system $(1,4)$.

Traditional treatments for renal cysts with symptoms include cyst aspiration (with or without sclerotherapy) and open/laparoscopic decortication of the cyst wall. Aspiration is the least invasive way, but it is less effective than open/ laparoscopic surgery. Laparoscopic decortication has been clinically proven to be more effective than aspiration and less invasive than open surgery, but multiple incisions and dissections are still inevitable. In this study, to combine the minimal invasiveness of aspiration and the maximum effectiveness of complete deroofing of a cyst in laparoscopic surgery, we resorted to the percutaneous nephrostomic decortication technique to treat simple renal cysts in the selected patients.

\section{Methods}

\section{Patients characteristics}

From November 2016 to January 2019, 18 patients (10 men and 8 women) who had simple renal cysts larger than $5 \mathrm{~cm}$, with or without abdominal pain, were evaluated via ultrasonography and contrast-enhanced computer tomography (CT). The mean (range) maximum diameter 
Table 1 Patients characteristics

\begin{tabular}{|c|c|}
\hline Parameters & Number or mean (range) \\
\hline \multicolumn{2}{|l|}{ Sex } \\
\hline Male & 10 \\
\hline Female & 8 \\
\hline Age, years & 48 (32 to 74$)$ \\
\hline Body mass index, $\mathrm{kg} / \mathrm{m}^{2}$ & 22 (19 to 28$)$ \\
\hline \multicolumn{2}{|l|}{ Side } \\
\hline Right & 11 \\
\hline Left & 7 \\
\hline \multicolumn{2}{|l|}{ Cyst location } \\
\hline Posterior upper & 5 \\
\hline Posterior middle & 9 \\
\hline Posterior lower & 4 \\
\hline Maximum diameter of cyst (range), $\mathrm{mm}$ & 58.5 (50 to 82$)$ \\
\hline \multicolumn{2}{|l|}{ Bosniak classification } \\
\hline I & 17 \\
\hline II & 1 \\
\hline \multicolumn{2}{|l|}{ Clinical presentation } \\
\hline Flank or abdominal pain & 8 \\
\hline Hypertension & 2 \\
\hline Parenchymal compression & 8 \\
\hline
\end{tabular}

of cysts was 58.5 (range, 50-82) $\mathrm{mm}$. All of the cysts were either located at or near the posterior part of the kidney to ensure the direct puncture into the cyst roof. Eight patients were admitted with mild flank or abdominal pain. Two patients were asymptomatic, but their cysts were larger than $6 \mathrm{~cm}$, and secondary hypertension was suggested. Eight patients had obvious renal parenchyma compression. Patients with Bosniak classification III cysts were excluded. Patients with multiple cysts and history of ipsilateral renal surgery were also excluded. The patients' demographic data are summarized in Table 1.

The present study was approved by the Ethic Committee of Xiangya Hospital of Central South University. All procedures performed in studies involving human participants were in accordance with the ethical standards of the institutional and/or national research committee(s) and with the Helsinki Declaration (as revised in 2013). Written informed consent was obtained from the patient.

\section{Surgical procedure}

All of the patients underwent general anesthesia. The patients were put in a prone position. A puncture was made into the cysts through the 11 th intercostal space or subcostal space under ultrasound guidance (Figure 1A), Puncture point was selected according to the location of the cyst to ensure getting into the cyst at the thinnest part of the cyst wall. Cysts' fluids were aspirated for cytological test. A guidewire was inserted through the 18-gauge needle into the cyst cavity. After this, dilation was made in sequence with fascial dilators from $8 \mathrm{~F}$ to $18 \mathrm{~F}$. An $8 / 9.8 \mathrm{~F}$ semirigid ureteroscope or $12 \mathrm{~F}$ nephroscope (Karl Storz, Germany) was used to examine the cyst wall with normal saline irrigant dripping from the height of $50 \mathrm{~cm}$. The entire inner wall of the cyst was inspected (Figure 1B), and then the sheath and nephroscope were retracted just outside of the renal cyst. In the plane between the cyst roof and perirenal adipose, the scope together with the sheath was used as the detacher to separate the cyst roof and the perirenal adipose (Figure 1C). In this process, we used the relative soft sheath, and not the irrigation nor the scope (Figure 1D). After the cyst roof was completely disassociated, another puncture was made, and an $8 \mathrm{~F}$ steel sheath was inserted into the space between the cyst and perirenal adipose space in the same incision (Figure 1E). A ureteroscopic forceps was inserted through the $8 \mathrm{~F}$ sheath to grasp the cyst wall, under the guidance of the scope, and gently pulled the cyst wall to a direction to keep tension within the cyst wall. Thulium or Holmium Laser were used to resect the cyst wall (Figure $1 F$ ). The fringe of the cyst wall was cauterized after the resection (Figure 1G). The resected cyst wall was sent for pathological exam (Figure $1 H$ ). During the surgery, the $8 \mathrm{~F}$ and $18 \mathrm{~F}$ sheaths were inserted in the same incision, (Figure 1I), which is about $1 \mathrm{~cm}$. A 16F drain tube was then placed in the retroperitoneal space after the procedure (Figure 17). All patients were discharged 1-2 days after the surgery. The tube was removed before discharge. All of the patients underwent bilateral kidney ultrasonography and at least one CT scan during the follow-up.

The brief surgical procedure is shown in Video 1.

\section{Results}

The percutaneous nephrostomic decortication (PCND) operations were successfully performed in all the patients. No major complications, such as active bleeding, urinary leakage or injury of the renal parenchyma or adjacent 

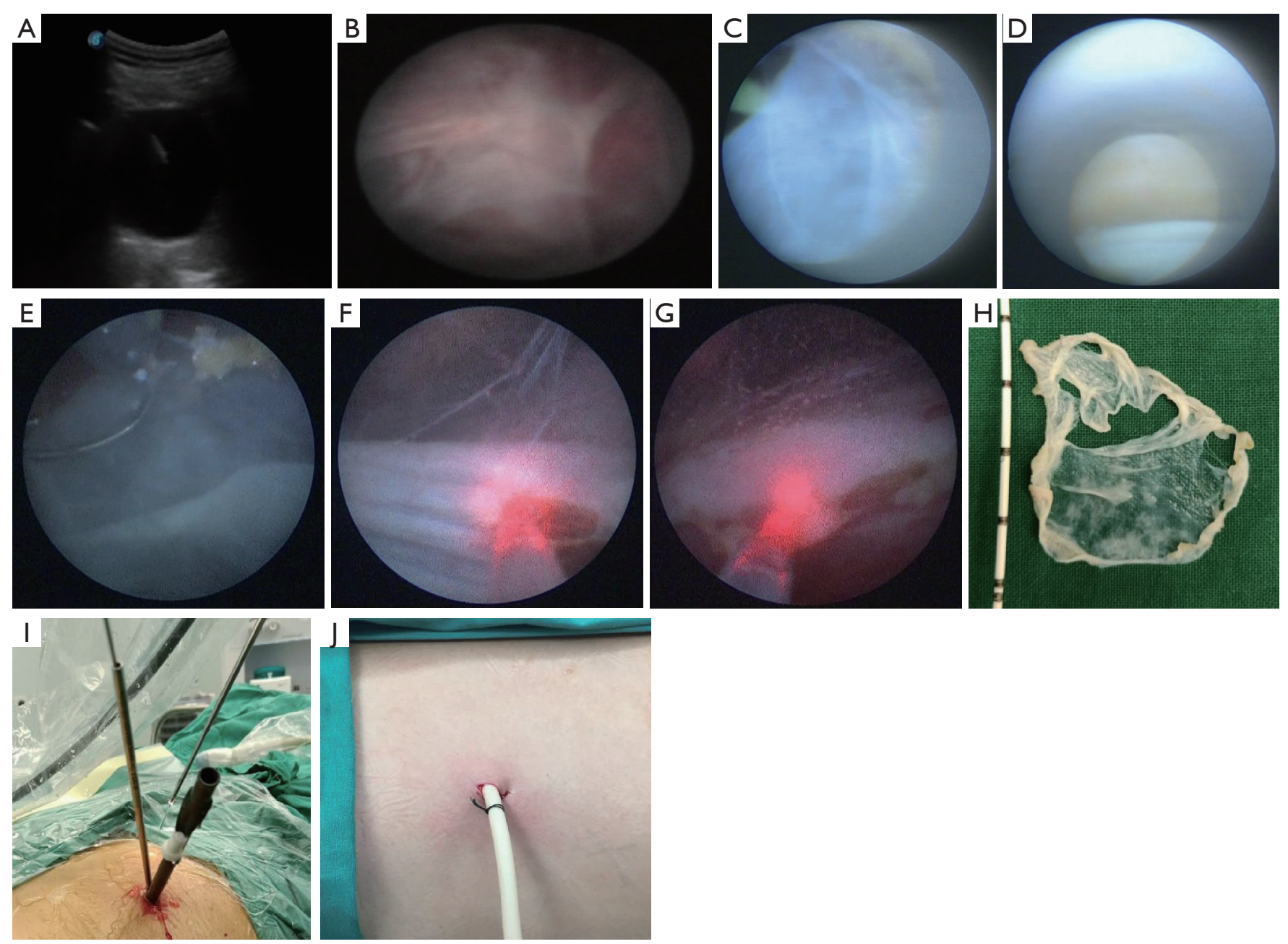

Figure 1 The process of surgical procedure. (A) ultrasound guided puncture to the cyst; (B) cyst inner wall examination; (C) finding the plane between the cyst wall (black arrow) and the perirenal adipose (white arrow); (D) cyst wall dissociation using the sheath; (E) the second steel sheath was inserted through the guide wire; (F) laser cutting of the cyst wall; (G) cauterization of the cyst fringe; (H) resected cyst wall; (I) two sheaths in the same incision; (J) $16 \mathrm{~F}$ drainage tube.

organs, occurred. Some patients had mild pain in the first day after the operation, but required no analgesic treatment. The mean operative time was 56.8 minutes (range, 38 to 115 minutes). The operative time was recorded as the time from the initial ultrasound-guided puncture to the placement of the retroperitoneal drain tube. The cysts' fluids cytology was negative for malignancy, and the histopathologic diagnosis of all cyst walls was consistent with simple renal cysts. The patients had a 3-month follow up after the surgery and once a year thereafter. Mean follow-up time is 20.6 months (range, 14-37 months). All the patients underwent bilateral kidney ultrasonography and at least one CT scan during their follow-ups. Of the 18 cyst units,
17 cyst units (94.4\%) were completely resolved with almost $100 \%$ disappearance of the cyst (Figure 2). One patient had a residual cyst which was reduced in size by more than $60 \%$. This patient didn't receive additional treatments during the follow-up. One patient had a small hematoma $(1.5 \mathrm{~cm}$ $\times 1.5 \mathrm{~cm})$ in the location of pre-surgery cyst without any symptoms and the hematoma was absorbed soon after. No edema was observed after the surgery; likewise, no obvious changes of serum $\mathrm{Na}^{+}, \mathrm{K}^{+}$concentration were detected.

\section{Discussion}

Simple renal cysts are common in the general population 

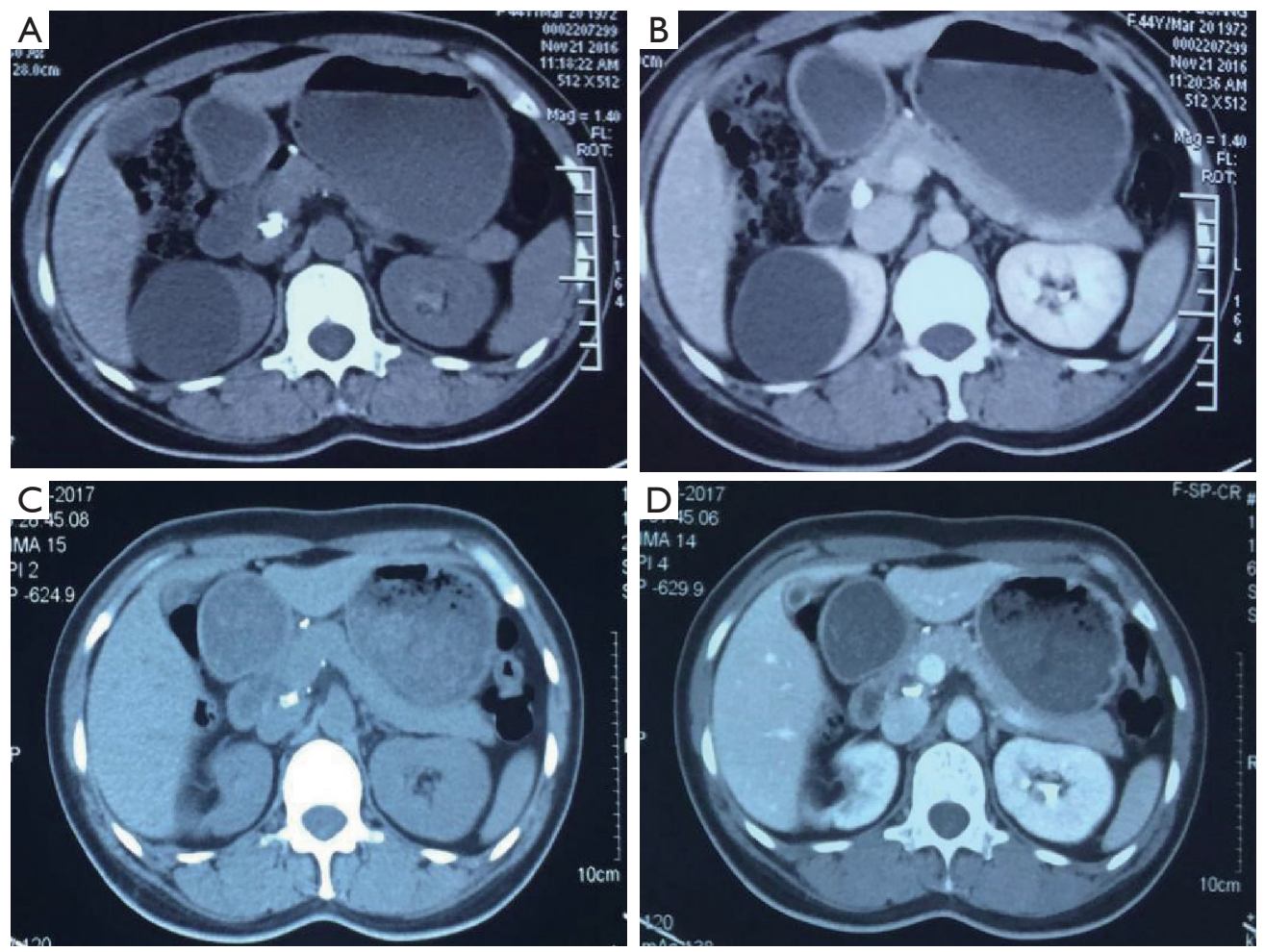

Figure 2 Before surgery and 1-year post-surgery CT comparison in the completely resolved patients. (A,B) CT scan before surgery; (C,D) CT scan 1-year post-surgery shows complete disappearance of the cyst.

and are usually asymptomatic. Generally, most renal cysts grow slowly and no surgical management are needed for those asymptomatic cysts. Pain, infection, parenchyma compression and collecting system obstruction are the major indications for surgical intervention $(3,5)$. However, when the diameter of the cyst is larger than $5 \mathrm{~cm}$ and renal parenchyma (collecting system) compression is present, regardless of the presence or the absence of flank or abdominal pain, intervention is required.

Nowadays, main choices of invasive intervention for renal cysts are percutaneous aspiration, either with or without sclerotherapy, and laparoscopic cyst decortication $(6,7)$. Laparoscopic decortication surgery has been proved to be an effective and durable treatment for symptomatic renal cysts (8-10). Percutaneous aspiration with or without sclerotherapy is the least invasive management for simple renal cysts. Reported Success rate is around $80 \%$ (11,12). Bas et al. (13) reported 184 patients with symptomatic simple renal cysts, 149 cases were treated with transperitoneal or retroperitoneal laparoscopic decortication and 35 cases with aspiration-sclerotherapy using 95\% Ethanol. The symptomatic and radiologic success rates for laparoscopic decortication group are $92.6 \%$ and $96.6 \%$ respectively, and for percutaneous aspiration sclerotherapy group, they are $54.2 \%$ and $60 \%$ in turn. Follow-up period is approximately 35 months. Complete cyst deroofing is the key process for laparoscopic surgery and also the main reason for its better outcomes than percutaneous aspiration. Clinical follow-up also shows that patients treated with aspiration and sclerotherapy had a much higher incidence of pain recurrence than laparoscopic treated patients (14).

With all the durable effectiveness and the minimal invasive process, laparoscopic surgery still needs three 1$2 \mathrm{~cm}$ incisions or a one 4- $\mathrm{cm}$ incision in Laparo-endoscopic single-site surgery. Laparoscopic surgery also has the risk of hypercarbia and intra-abdominal organ injury. during a transperitoneal approach, laparoscopic surgery also has the risk of postoperative ileus, and hernia formation (15), so it is more invasive with a better outcome compared to percutaneous aspiration.

Percutaneous nephrolithomy is widely used to treat renal stone in the world. Many urologists had tried the percutaneous approach to treat renal cysts. In 1990, Gelet et al. (16) first reported six cases of simple renal cysts 
treated with the percutaneous deroofing surgery using a transurethral resectoscope to cauterize the cyst wall. Plas et al. (17) performed 10 cases of percutaneous resection of renal cysts from 1985 to 1991 . They had used a standard $22 \mathrm{~F}$ resectoscope to resect the peripheral cyst wall from the inside out. Median follow-up time is 45.7 months (range, 26-66 months). Results showed that 5 patients had no relapse, 2 patients had residual cysts. The other 3 patients had newly developed cysts and were thought to be genuine recurrence after primary successful treatment. All of the 10 patients were symptom-free after surgery. Although it seems that percutaneous process is less invasive, there were few reports about the percutaneous deroofing process to treat the renal cysts since then. The reason may be its difficulty and sometimes the risk of injuring the adjacent organ, for the doctor to completely resect the roof of the cysts using the transurethral resectoscope in the retroperitoneal space, especially when they did the resection inside-out without dissociating the cyst wall from the perirenal adipose.

During our first two PCND surgeries, we found it very difficult to both simultaneously have a clear view of the retroperitoneal space and to hold tension within the cyst wall for laser cutting. Because the membrane of the cyst was pulled toward the scope by the forceps in the same F18/F20 tract, we had to cut the roof in small pieces within the narrow sheath. To solve this problem, in the following cases, we put in another $8 \mathrm{~F}$ steel sheath just beside the $18 \mathrm{~F}$ Amplazt sheath in the same $1 \mathrm{~cm}$ incision. In this sheath we put in an ureteroscopic forceps and pulled the cyst wall to a proper direction to keep tension within the cyst wall for laser cutting without obstructing the view of the scope. With this little improvement, we could cut the whole cyst roof in one piece, just like the laparoscopic surgery, and the operation time got nearly reduced by half.

We use Thulium and Holmium laser as cutting tools. From our experience, Thulium laser is far more efficient in cutting the thin membrane of the cyst. The Holmium laser is a pulse laser, which tends to make a succession of holes in the membrane, and sometimes you have to use the forceps to detach it from the kidney. Thulium laser has a very good cutting and hemostasis efficiency in this process (18).

We used ureteroscope or 12F nephroscope in the surgery. There is no obvious difference between those two scopes in the process. Since we used saline dripping irrigation from the height of $50 \mathrm{~cm}$ instead of pump, the water pressure in the retroperitoneal space was very low. There was no edema observed after the surgery, nor any obvious changes in patients' serum $\mathrm{Na}^{+}, \mathrm{K}^{+}$concentration.
In the percutaneous surgery, the resection region of the cyst roof is the same as the traditional laparoscopic approach, but the incision of PCND surgery is about $1 \mathrm{~cm}$ and shows superior cosmetic results than laparoscopic surgery in renal cyst patients. We suggest that it will have a similar recurrence rate as laparoscopic surgery.

With all of its minimal invasiveness and good results, the shortcomings of our PCND procedure are also obvious. Poor vision and operating space in the surgery are the most common inconveniences for the surgeon. The sheath was used to detach the cyst wall from the perirenal adipose, and during this process, the surgeon should be cautious to begin with the proper plane to detach the adipose in a whole piece. If the adipose is dissected and broke into pieces, it will be very difficult to have a clear retroperitoneal space. For the same reason, overweight patients are not a good candidate for PCND. A longer skin to cyst distance may increase the difficulty of the puncture. Thus, reducing the accuracy of it.

The other shortage of PCND procedure is its cutting tools. In some patients, the cyst wall will become relatively thick when the cyst fluid is sucked out. It will be very difficult to avoid bleeding using Holmium laser. Once the cyst wall begins to bleed, complete roof decortication will become a very difficult task because of poor vision. Post operational CT scan shows that the only patient who had a residual cyst also had a very thick cyst wall post-surgery (Figure 3). In that condition, Thulium laser may be the better choice. Because the puncture to the cyst must be made through the thin roof of the cyst to avoid bleeding, PCND surgery is not suitable for the cysts which are completely anterior nor for patients with a history of kidney surgery.

Percutaneous nephrostomic decortication has proved to be both an effective and a minimally invasive surgical procedure for simple posterior renal cysts in selected patients. Urologists with experience in percutaneous nephrolithotomy under sonographic guidance can perform this surgery without a special training. Given that our study used a relatively small patient sample and lacked a control group, thus, randomized studies with larger cohorts are required to compare the safety and efficacy of both PCND and laparoscopic procedures for the treatment of renal cysts in the future studies.

\section{Acknowledgments}

We thank patients who participated in the study. And thank 

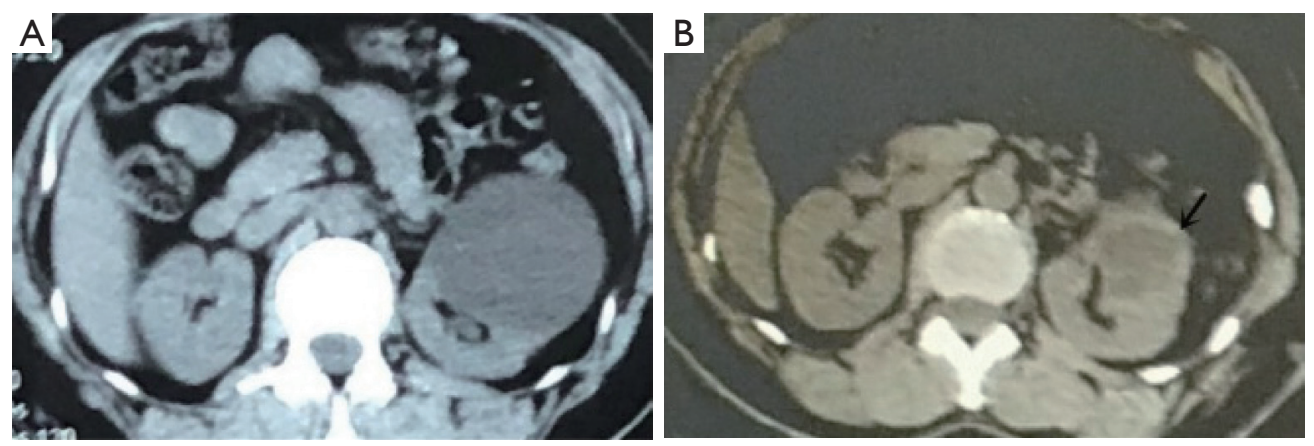

Figure 3 Before surgery and post-surgery CT comparison in the residual cyst patients. (A) CT scan before surgery; (B) post-surgery CT scan shows that the remaining cyst wall is very thick (black arrow).

Dr. Belaydi Othmane for revising the manuscript.

Funding: This study was supported by the National Natural Science Foundation of China 81770705 (to H Chen) and Natural Science Foundation of Hunan Province 2020JJ4871 (to Z Chen).

\section{Footnote}

Conflicts of Interest: All authors have completed the ICMJE uniform disclosure form (available at http://dx.doi. org/10.21037/tau-20-875). The authors have no conflicts of interest to declare.

Ethical Statement: The authors are accountable for all aspects of the work in ensuring that questions related to the accuracy or integrity of any part of the work are appropriately investigated and resolved. The present study was approved by the Ethic Committee of Xiangya Hospital of Central South University. All procedures performed in studies involving human participants were in accordance with the ethical standards of the institutional and/or national research committee(s) and with the Helsinki Declaration (as revised in 2013). Written informed consent was obtained from the patient.

Open Access Statement: This is an Open Access article distributed in accordance with the Creative Commons Attribution-NonCommercial-NoDerivs 4.0 International License (CC BY-NC-ND 4.0), which permits the noncommercial replication and distribution of the article with the strict proviso that no changes or edits are made and the original work is properly cited (including links to both the formal publication through the relevant DOI and the license). See: https://creativecommons.org/licenses/by-nc-nd/4.0/.

\section{References}

1. Skolarikos A, Laguna MP, de la Rosette JJ. Conservative and radiological management of simple renal cysts: a comprehensive review. BJU Int 2012;110:170-8.

2. Rané A. Laparoscopic management of symptomatic simple renal cysts. Int Urol Nephrol 2004;36:5-9.

3. Hemal AK. Laparoscopic management of renal cystic disease. Urol Clin North Am 2001;28:115-26.

4. Weichert-Jacobsen K, Loch T, Küppers F, et al. Clinical experience with percutaneous renal cyst resection. BJU Int 1999;84:164-6.

5. Agarwal MM, Hemal AK. Surgical management of renal cystic disease. Curr Urol Rep 2011;12:3-10.

6. Camargo AH, Cooperberg MR, Ershoff BD, et al. Laparoscopic management of peripelvic renal cysts: University of California, San Francisco, experience and review of literature. Urology 2005;65:882-7.

7. Li YZ, Li MX, Wang T, et al. Efficacy and safety of alcohol sclerotherapy involving single-session multiple injections to treat simple renal cysts: a multicenter, prospective, randomized, controlled trial. Chin Med J (Engl) 2013;126:803-7.

8. Pearle MS, Traxer O, Cadeddu JA. Renal cystic disease. Laparoscopic management. Urol Clin North Am 2000;27:661-73.

9. Yang D, Xue B, Zang Y, et al. A modified laparoendoscopic single-site renal cyst decortication: single-channel retroperitoneal laparoscopic decortication of simple renal cyst. J Laparoendosc Adv Surg Tech A 2013;23:506-10.

10. Atug F, Burgess SV, Ruiz-Deya G, et al. Long-term durability of laparoscopic decortication of symptomatic renal cysts. Urology 2006;68:272-5.

11. el-Diasty TA, Shokeir AA, Tawfeek HA, et al. Ethanol 
sclerotherapy for symptomatic simple renal cysts. J

Endourol 1995;9:273-6.

12. Mohsen T, Gomha MA. Treatment of symptomatic simple renal cysts by percutaneous aspiration and ethanol sclerotherapy. BJU Int 2005;96:1369-72.

13. Bas O, Nalbant I, Can Sener N, et al. Management of renal cysts. JSLS 2015;19:e2014.00097.

14. Okeke AA, Mitchelmore AE, Keeley FX, et al. A comparison of aspiration and sclerotherapy with laparoscopic de-roofing in the management of symptomatic simple renal cysts. BJU Int 2003;92:610-3.

Cite this article as: Chen H, Li Y, Zeng F, He C, Cui Y, Chen J, Zeng H, Lu S, Wang W, Chen Z. Percutaneous nephrostomic decortication: a microinvasive surgery for posterior renal cyst. Transl Androl Urol 2020;9(6):2764-2770. doi: 10.21037/tau-20-875
15. Ferzli G, Raboy A, Kleinerman D, et al. Extraperitoneal endoscopic pelvic lymph node dissection vs. laparoscopic lymph node dissection in the staging of prostatic and bladder carcinoma. J Laparoendosc Surg 1992;2:219-22.

16. Gelet A, Sanseverino R, Martin X, et al. Percutaneous treatment of benign renal cysts. Eur Urol 1990;18:248-52.

17. Plas EG, Hübner WA. Percutaneous resection of renal cysts: a long-term follow-up. J Urol 1993;149:703-5.

18. Tontini GE, Neumann H, Pastorelli L, et al. Thulium laser in interventional endoscopy: animal and human studies. Endoscopy 2017;49:365-70. 